\title{
Transformative and Innovative E-Gov for the Next Generation: Linkages of Back Offices for One-Stop Portal
}

\author{
Osamu Sudoh ${ }^{1,3}$ and Yumiko Kinoshita ${ }^{2,3}$ \\ ${ }^{1}$ Professor, ${ }^{2}$ Assistant Professor, \\ ${ }^{3}$ Graduate School of Interdisciplinary Informatics, the University of Tokyo \\ 7-3-1 Hongo Bunkyo-ku Tokyo 113-0033 \\ \{Sudoh, kinoshita.yumiko\}@iii.u-tokyo.ac.jp
}

\begin{abstract}
It is imperative that e-Gov platform for the next generation achieves integrative administrative services via citizen's one-stop portal and back-office linkages among ministries and external organizations. This objective aims to make further advancement in system integration and coordination by employing cloud computing and service-oriented architecture (SOA). To achieve these goals, expertise knowledge must be provided as well-organized citizen services, for which e-Gov platform should be built upon loose-coupling of databases. Japan's e-Gov policy has addressed these needs and designed its national service platform using master registry for standardized metadata, which is the first attempt in the world. This paper discusses the objectives of Japan's e-Gov policy, the architecture of one-stop service platform, and its evolutionary process of back-office linkages using common registry. It is proposed to manage metadata in labor saving manners according to a matrix of operation and disclosure classes via pre/post-process filters assigned.
\end{abstract}

Keywords: e-Gov, one-stop service, loose coupling, public innovation, XML.

\section{Next-Generation e-Gov Platform for Japan}

One of critical tasks in establishing e-Gov platform for the next generation is to achieve integrative administrative services via citizen's one-stop portal and back-office linkages among ministries, agencies and external organizations. This objective is pursued by further advancement in inter-governmental and inter-organizational coordination by employing cloud computing and service-oriented architecture (SOA), for strategic challenges i.e. the support of private enterprises including smaller firms [1], the facilitation of labor market to face employment issues of today [2], the reform of policies for the aging society [3] and the promotion and utilization of environmental protection technologies [4]. To achieve these goals, expertise knowledge must be provided as well-organized services to citizens with advanced information technologies (IT). For these purposes, e-Gov platform should be built upon loose-coupling of data, which are currently owned by individual institutions. Japanese government has addressed the 
need of database linkages in e-Gov and designed its national service platform using service hub regulating registry, directory and operation processes.

Strategic Headquarters for the Promotion of an Advanced Information and Telecommunications Network Society, established in Japan's Cabinet Office, presented a report entitled ' $i$-Japan Strategy 2015' in July 2009 [4]. The report elaborated on priority areas for Japan's e-Gov initiative such as further utilization of IT in healthcare, revitalization of industry and local communities and nurturing of new industries, and improvement of digital infrastructure. These efforts are imperative so that Japanese industries continue to innovate e-Commerce and management system, implement Business Process Reengineering (BPR), for all sizes of enterprises, and gain competitiveness in global markets, strengthening safety measures, and integrating digital technologies in production and delivery of services for enhanced value-added products. According to these directions, the government supports the digitization of public and administrative information as well as the reform and optimization of administrative processes and systems by designing e-Gov and e-Municipality platform based on cloud computing system to integrate servers and databases owned by administrative agencies. National and local governments are also encouraged to implement BPR, and as a part of their optimization efforts, the use of National e-Post Office (P.O.) Box, a type of repository for citizens, is emphasized to reduce administrative costs substantially ${ }^{1}$. They will allow for the facilitation of social security reform and the provision of a wide range of services.

In October 2007, Project Team for the Next Generation e-Gov and Public Services, chaired by Osamu Sudoh (the author), was launched in Japan's Cabinet Office to discuss technical directions to implement full-fledged one-stop service for citizens. In October 2009, a report on the next generation e-Gov system and one-stop service published was summarized [5]. This report specified design, methodology, and technical specifications for back-office linkages with several attempts leading to the first-ever common registry and directory across organizations. In line with this report, this paper discusses the objectives of Japan's e-Gov policy for the next generation, the architecture of one-stop service platform, and the process of back-office linkages. As for the incorporation of the registry, also to be prepared for virtualization technology of future enhanced networks and security, it is proposed to manage information in cost and labor saving manners according to a matrix of operation and disclosure class via pre- and post-process filters and XML schemes.

\section{Needs for Integration and Linkages of Back Offices in e-Gov}

Sudoh, Inoue, and Nakashima [6] articulate the issue of aging society, and is developing preventive healthcare system utilizing IT as a part of Japan's e-Gov initiative. Various research projects for preventive medicine utilizing IT are currently underway in the world. Pfizer and Grameen Health $(\mathrm{GH})$ announced a partnership for healthcare

\footnotetext{
${ }^{1}$ The report expects for a reduction of costs by $30 \%$ by the introduction of e-PO Box.
} 
delivery and financing for the exchange of expertise knowledge, and the provision of micro-health insurance, telemedicine and mobile healthcare ${ }^{2}$. These developments can be called service innovation as they represent a new combination [7] of technologies, new services, means of delivery, and social infrastructure.

In line with urgent social and economic needs for the next generation, European Union, for instance, released a report on e-Gov strategy [2] focusing on 'a single information market enabling cohesion and growth.' An important aspect of this direction is that enterprises and institutions, when they are capable of accumulating high-level expertise and produce enhanced value added, may maintain a closed environment to generate expertise knowledge while they can choose to open themselves for partnership and outsourcing to access external sources for information services. Is the next generation e-Gov platform designed to standardize knowledge, or to generate capacity to allow for the diversification of knowledge through the implementation of cloud computing and SOA in and through public domains?

An immediate challenge for the e-Gov initiatives is cost sharing with business sectors and external organizations, which have grown substantially through the implementation of IT and investment into human capital. Furthermore, it is important to provide education and secure skilled human resources in service sectors, which share a large part of employment in today's economy. The idea of using knowledge-intensive services for providing high-quality public services is being presented for the development of skilled human capital and acquisition of external knowledge. Inter-overnmental e-Gov initiative is also promoted to share information on employment for higher mobility of workers [8].

To provide citizens with high-quality services to achieve service innovation in society and economy, administrative agencies may face challenges in building cooperation and partnerships with private firms because of knowledge and technological intensity inherited in such services. Therefore, it is important to incorporate loose-oupling of databases owned by multiple organizations based on advanced service technologies. Furthermore, cloud computing is currently recognized as 'information utility,' and the balance of public cloud and private cloud must be considered carefully in terms of Hardware-as-a-Service (HaaS), Platform-as-a-Service (PaaS), and Software-as-a-Service (SaaS) so that differentiation and competitiveness of services are achieved in data specifications and database linkages. (Please refer to Rimal, Choi, and Lumb [9] and Zhang and Zhou [10] for a good survey and description of cloud computing system).

Information utility means that customer interacts directly with a central computer and information files from a remote terminal [11]. Therefore, network-based services are offered to both small and large companies. For instance, Google's Bigtable is de-

\footnotetext{
${ }^{2}$ Micro-health is a low cost health insurance for i.e. one dollar per person a year. Bellinghen, D.V.: Grameen Health and Pfizer Announce Novel Partnership To Explore Sustainable Healthcare Delivery Models for The Developing World, Corporate news (September 24, 2008), http://www.pfizer.be/Media/Press+bulletins/Philantropy/Grameen+Health+and+ Pfizer+Partnership.htm

${ }^{3}$ The report mentions that 'key issues are addressed as the realization by Member States of the value of a single market in information and communication technologies (ICT).' http://ec.europa.eu/information_society/eeurope/i2010/greenknowledgesociety.pdf
} 
signed to handle a mixture of structured data and unstructured data [12] for the use of home networking and a variety of terminals [11]. Therefore, Bigtable currently allows clients to set up 'lazy replication between their tables (cross-data-center replication)' [12]. In the future, it is likely and possible to name information by itself, achieving object-to-object connectivity, object storage and retrieval through name resolution and routing by bringing a new abstraction technique [13]. Such a large scale database is adopting expressive queries to describe server-side filtering of data [12]. On the other hand, specifications of data are an important area for us to elaborate when cloud computing is introduced in private and public domains while some of current cloud systems may not employ SOA yet. In this case, cloud computing should deploy novel data management approaches, such as analytical data management tasks, multi-tenant databases for SaaS, and hybrid designs among database management systems (DBMSs) [14].

To achieve one-stop services for citizens, SOA-based system and other types of systems must be coordinated, and deployed over new architectures. Denmark summarized a report in 2004, in which 2500 examples of e-Gov projects in EU were surveyed to find what constitutes better e-Service in public administrations. Good public service requires database linkages by establishing a system for coordinating multiple and simultaneous service requests ${ }^{4}$, one-stop portals, proactive and self services, and agent-based services [15]. To do so, it says that workflows of administrative operations and processes must be aligned well, and common service modules must be increased. It is also important to help users to cope with complex legislation and procedures requiring expertise rarely possessed by the average citizens. e-Gov strategy for the next generation must meet these technological, socio-economic, and democratic challenges in the delivery of one-stop services $[4,5]$. The following sections deal with e-Gov initiative in Japan to discuss its objectives, challenges, approaches, and advantages.

\section{Providing Full-Fledged One-Stop Portal}

Project Team for the Next Generation e-Gov and Public Services in Japan's Cabinet Office presented technological approaches for one-stop portal in October, 2009 [5]. One-stop portal is a front-end of all public services for citizens, and is an integrative part of administrative service platform. Administrative services and applications will be coordinated in this one-stop portal. As Fig. 1 shows, Japan's one-stop portal provides easy to understand information for citizens, and agent-based services. The one-stop portal also responds to user's request for information through proactive service on website, provides customizing functions of webpage ${ }^{5}$, and helps intelligent search so that citizens can find information on administrative procedures and documents easily.

To link databases among multiple organizations, it is important to visualize current operation, process, and data. It is technically possible to achieve back-office linkages as long as databases are structured, metadata schemas are standardized, and the

\footnotetext{
4 Back-office clearing house corresponds to 'service hub' in Japan's e-Gov system, which is explained in detail in later sections.

5 The customization of webpage is the function to create so called 'My Page.'
} 
specifications of interface and conversion rules of codes and XML schemas are fixed. Then, cross reference of administrative information, location, access protocols, and data format is achieved for existing systems employing different technological specifications. To do so, however, it is not necessary to consolidate all administrative information in one location, but only metadata schemas should be processed at the central service hub in registry, directory, and process control. Therefore, back offices are to be loosely-coupled, which provides extensibility to designs.

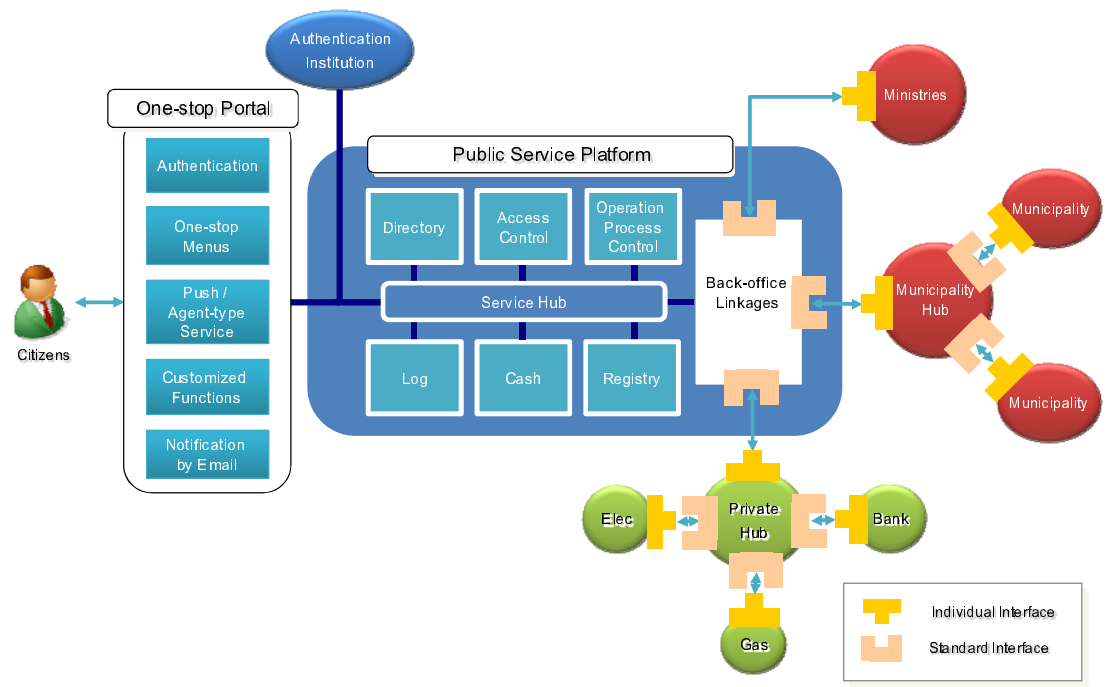

Fig. 1. Next-generation One-stop Portal for Japan's e-Gov [5]

Japan's e-Gov systems are categorized into legacy system ${ }^{6}$, local information platform $^{7}$, and SOA-based system. The public service platform must respond to distinct technologies employed for these different types of systems in e-Gov infrastructure. Therefore, as Fig. 2 shows, each external system must be filtered through a type of adopter (filter) for back-office linkages to minimize reconstruction efforts of existing systems and maximize the potential of service integration. Currently, the service hub has directory, registry, and operation process control functions. The directory designates a list of services and representative agencies, location IDs, and citizen's log-in IDs. The registry controls specifications of metadata and services. Operation process control function navigates citizen's request for services.

Administrative agencies and external organizations access to a collection of metadata, which is to be controlled in public service platform. For example, a local

\footnotetext{
${ }^{6}$ Legacy system refers to all systems that are difficult to get connected or coordinated with external systems. A large part of Japan's e-Gov systems are legacy enterprise system.

${ }^{7}$ Local information platform refers to operational and technical standard for municipalities' system. It governs specifications for multi-agency systems to facilitate inter-linkages of systems operated by national government, local governments, and private sectors.
} 


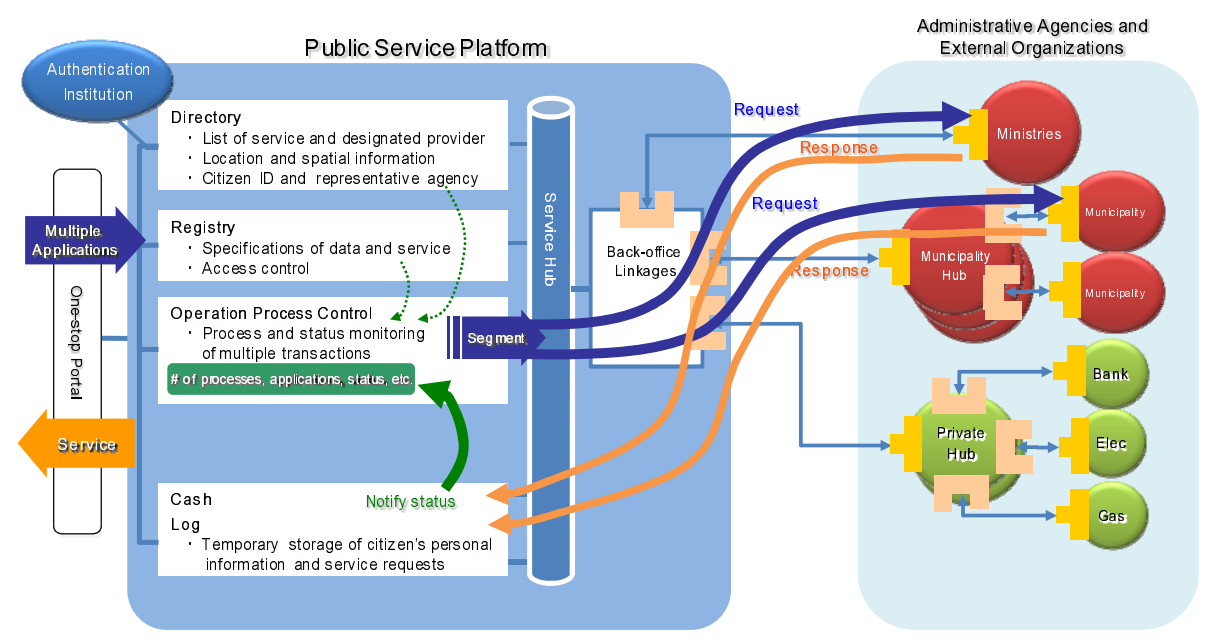

Fig. 2. Public Service Platform with Service Hub [5]

municipality's system is granted an access to a central server to reference metadata schemas in directory and registry, so that multiple applications from citizens are handled by operation and process control functions in coordination with several public and private systems, and authentication is granted in one process. Data format and access protocols are managed in the public service platform via the service hub. The server reduces information passed into a service to key data stored by national and local governments' enterprise systems. This mechanism of database linkages is gathering attention, not only from government agencies, but also as a collaboration platform for public, private and academic institutions.

When a citizen accesses the one-stop portal, the operation process control finds a list of services to be processed. The directory provides addresses of representative agencies while the registry defines service specifications. Other citizen's information is obtained from the log. All procedures go through the filter to get to the external systems. Corresponding external systems return services to be stored temporarily in cash, and the operation process control function notifies the citizen of the result of his or her requests at one time. Therefore the process control function handles a story space i.e. story, actor, scenario, and context in this web information system.

\section{Achieving Back-Office Linkages}

\subsection{Goals and Challenges}

To achieve the consolidated one-stop service and back-office linkages, metadata characterization should be defined in the registry and directory to retrieve data from multiple locations. Then, public information must be digitized and disclosed according to pre-specified protocols and mechanisms to make them available for citizens. Data are currently controlled by each administrative agency and external organization, but in 
principle they should be accessible for citizens according to a unified standard procedure. In Switzerland, Document Management System (DMS) started in 2007 in an organizational unit called Competence Center (CC) to manage all government documents on web-based collaboration platform, and avoid distributing documents in print or email [16]. Chinese e-Gov, for example, designed data storage utilizing ETL (extraction, transformation and loading) technique by using dimensional data in a star structure [17]. It is also proposed to create metadata table and subscribe information of users [18].

The standardization of all metadata schemas in XML may give huge burdens on public agencies and private organizations to restructure databases particularly when several distinct systems are being employed. In addition, the configurations of XML schemas to link existing relational and object-based databases would require constant updates of themselves as new services are added to the central service hub ${ }^{8}$. Besides, the disclosure of personal information and the standardization of all service processes and operations are currently not realized. In such a situation, standardized term tables and data definitions need to be revised and updated constantly by each agency and organization as their meaning and usage are under unintermittent pressure for change so that the quality of public services is maintained and improved. In addition, to provide customization functionality for the portal i.e. mash-up and access to external contents, information extraction techniques ${ }^{9}$ are necessary, for example, the use of hidden tables in web pages to induce entity relationships [12], statistical entity extraction [19], entity disambiguation using Markov Logic Networks, and semantic web with cloud computing [20].

It should be noted that the server will be capable of extracting XML schemas and entity relationships, or interpret entities from unstructured data. It is also supposed that the public service platform becomes open to the adoption of third-party applications for the promotion of BPR. Virtualized networks would access public information directly through the filter ${ }^{10}$. With these challenges behind, the service platform is designed to have conversion libraries ${ }^{11}$ for Japanese letters and characters as well as pivot tables for

\footnotetext{
${ }^{8}$ According to Ma et al. [21] who surveyed more than 8,500 database schemata, even developers would feel hard to work with 20 or more relationships. SAP/R3's relational schema contains more than 21,000 tables.

${ }^{9}$ Information extraction techniques are used at entity-level, sentence-level, and page or corpus-level. Statistical method can be embedded into the filter after the linkage of back offices would reach a certain level of coordination; however, existing technologies for information extraction normally require machine learning or human annotation to achieve a good analysis of dependencies. It is possible that the current public websites, user's request history, and help files are used to extract important entity or object metadata, and use them as metadata characterization.

${ }^{10}$ The filter handles constraints for the registry such as component construction (based on existence and inclusion of components), identification (the constructor of a set), equality generation (for a set of objects from one class or from several classes), object generation, and representation of constraints through structures [21].

${ }^{11}$ Legacy systems need extra systems for database linkages such as datastore, a conversion filter, and directory for ID conversion. Systems based on local information platform do not necessarily need these systems. SOA-based advanced systems are capable of achieving semi-automatic linkages. Therefore, it is supposed that SOA-based systems are suitable for operation and process optimization.
} 


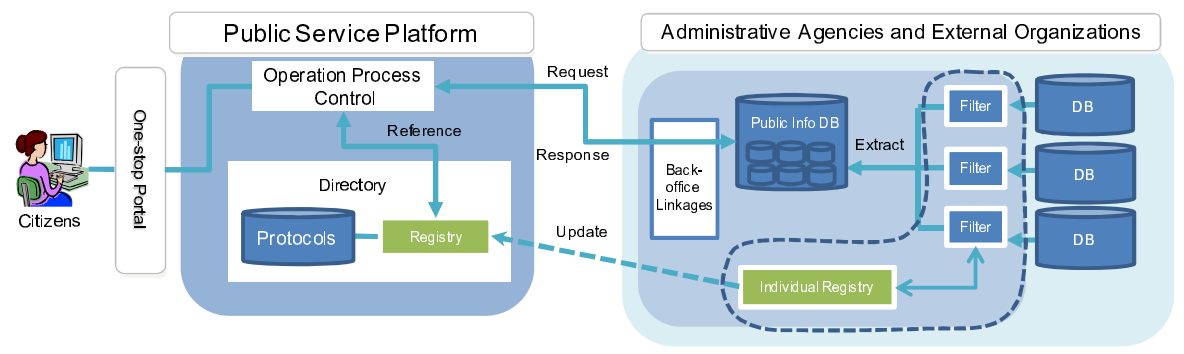

Fig. 3. Prerequisite Set-up for Back-office Clearing [5]

XML schemas to a certain degree (see Fig. 3). These conversion libraries are prepared as a set of standardized templates for all public services for the one-stop portal.

\subsection{Public Service Platform}

\section{Common Registry and Directory}

As previously mentioned, the loose-coupling of databases is proposed so that only the metadata is defined by datatype, attribute and character associated with public information, service processes, in which services of representative agencies are extracted into the public server and controlled by the public service platform. To enable these procedures, the registry and directory are designed as follows.

First of all, ministries and external organizations go through the filter to create a list of core terms in a table (See Fig. 4). Terms can be extracted from existing public websites, help files, and menu bars so that most commonly used terms and key terms are registered in the initial term table. The interface has a matching and pop-up function. A designated personnel in administrative agencies and external organizations uses the filter to feed information to the public service platform. The functionalities of the filter are to analyze documents and data provided against the term table and information that has already been supplied by the other agencies and organizations, prompt the personnel to apply necessary changes and modifications to data definitions according to a preset style guide (a set of rules on the use of character codes and letter sets, and grammatical guidelines at sentence and term levels), and assign ministry ID, department ID, personnel ID, original term ID, timestamp, multi-lingual ID, and other discretionary IDs. It also returns information quality analysis ${ }^{12}$ to the personnel for later changes and updates.

In this pre-process procedure, agencies and organizations specify operational and disclosure classes ${ }^{13}$ so that information and data are controlled according to a matrix of operation and disclosure protocols. The operation class refers to a stage of processes in

\footnotetext{
${ }^{12}$ The quality of feeding information can be scored by accessibility, security, representational quality i.e. ease of understanding, contextual quality, and concrete representation [21].

${ }^{13}$ Class-wise representation based on Entity-Relationship models uses classes, and object-wise representation do not use classes [22]; however, the registry and directory need these classes to direct multiple processes to multiple locations securely.
} 
a service i.e. life stages and workflow, lifespan of objects, loop and networked data, phases in the life cycle, recording of the development, enhancement, and ageing of objects [21]. Disclosure class is defined in standardized protocols regarding the level of disclosure of information for each service request. Each agency and organization does not reconstruct XML schemas for their databases, but the filter attaches XML, automatically or semi-automatically, with some inputs from the personnel which are prompted by the filter, and update history is logged so that the log can be used later to direct multiple service requests to multiple locations.

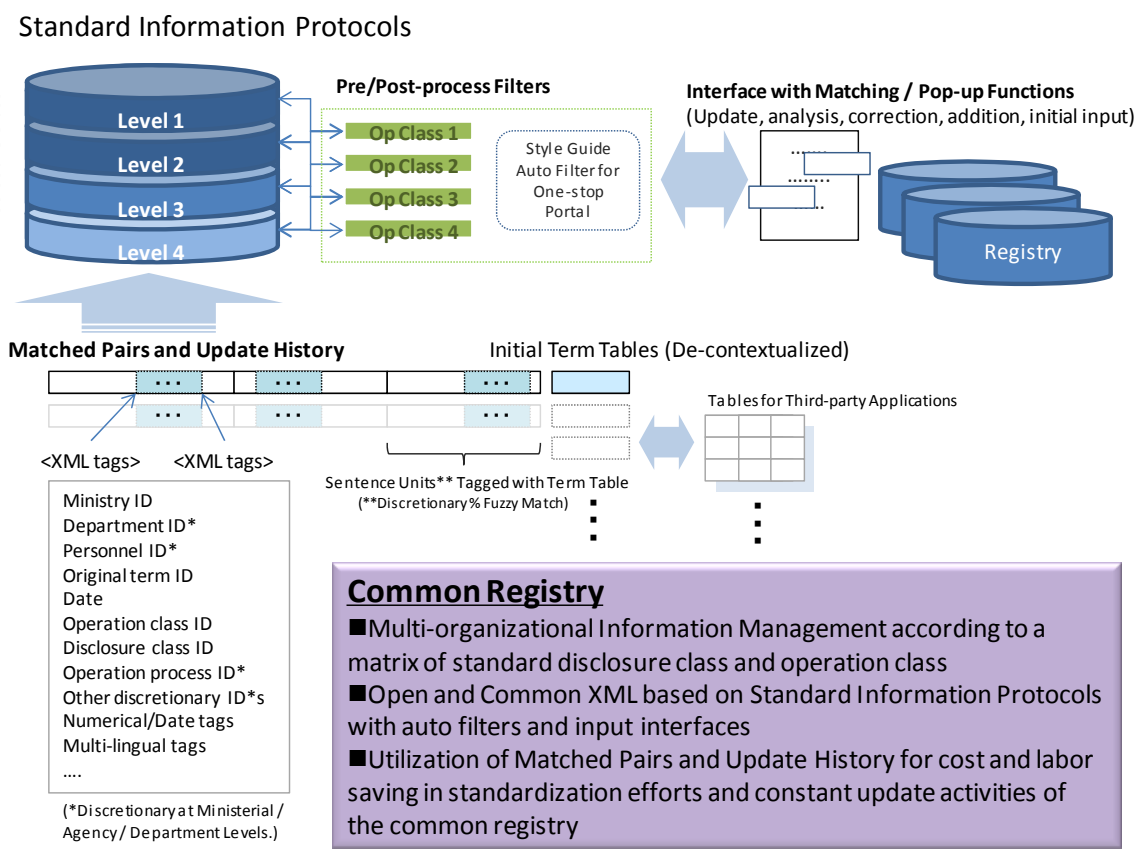

Fig. 4. Schematics of Registry and Directory

\section{Term tables and XML}

As previously described, the term table is the initial step to construct the registry and directory. The table works as a reference for agencies and organizations to feed data as a prerequisite step to launch the one-stop portal. The table should be a collection of common terms and key terms are registered without relational or object attributes i.e. utilization recording meta-structures, and quality meta-structures. Index, external keys, and object names and instances can be added to the term table when they are supplied from existing structured databases. The character conversion table is utilized in this process.

Once the list of core terms is created, the designated personnel access to the list and supply data from own databases. The interface requests automatically for necessary information, and the provided data is associated with a term or multiple terms to be 
stored at a sentence level ${ }^{14}$. Sentences are stored in matched pairs ${ }^{15}$. These data, then, represent meta-characterization in terms of insertion, update, deletion time, keyword characterization, utilization pattern, format descriptions, utilization restrictions, rights i.e. disclosure, obligations and costs, and technical restrictions. These data, when XML are inserted, represent temporal, spatial, ownership, representation, and context data [21]. The filter return an analysis report to the personnel according to these criteria so that the agency or organization can improve the information for later updates and changes ${ }^{16}$.

The data of matched sentences supplements the representation of associations of objects and entities, which are lack of in the information and data stored in the central registry and directory. (Please refer to Franceschet, Gubiani, Montanari, and Piazza [22] for more information on entity relationship and XML schema.) It is suggestive that the sentences should not be nested or linked in a star structure to avoid inconsistencies of meta structures. The directory references multiple sentence units for requesting multiple agencies and organizations to process a service request ${ }^{17}$. In such a case, the log obtained during the pre process procedure is also utilized. As the matched pairs are used more in the provision of one-stop services, meta-properties i.e. category, source, and quality information can be used to update the filter functionality so that the filter is able to give an analysis on usage, meta characterization, log dimension, etc. Simultaneously, the analyzed information should be used to update the term table so that terms, which are no longer used or not frequently used, are eliminated. It is also preferred that third-party applications are allowed to reference both the initial term table and matched pair histories according to a discretionary disclosure class assigned.

\section{Pre- and Post-process Filters}

When the citizen requests for a service, the operation process control references XML in the registry and calls for services from multiple locations according to the directory. In this process, the disclosure class ID and operation class ID are used to give multiple service requests to different systems. These processes go through the filter to get to

${ }^{14}$ The filter searches for a term to be associated with the supplied data at a sentence level. In this process, the sentence should contain an exact matched term, and in a 60-80\% matching range (or another discretionary matching percentage) at a sentence level. The minimum unit of association is $100 \%$ matched character sets. It gives easiness for the personnel, even when the personnel is not a technician, to review the information and add necessary updates. The maintenance of the matched pairs is extremely easy when there is a chance only in document version number, numerical expressions in a sentence, proper nouns, etc.

${ }^{15} \mathrm{XML}$, sentence pairs, and their update histories should be open to the other agencies and organizations for consistency and quality improvement across all agencies and organizations.

${ }^{16}$ It is possible to add steps later such as aims, purposes, and subjects i.e. actors involved, method and heuristics (i.e. specification language, and simplification approach), developed documents results (i.e. results, deliverables), enabling condition for step (i.e. gathering, dependencies, participation, etc), and termination condition for step (i.e. completeness, correctness, sign-off, contracts, quality, and obligation for the step fulfilled) [21].

${ }^{17}$ Circulation meta-structures for documents, for instance to display objects in different phases for legal documents, should be stored in databases controlled by agencies and organizations. 
individual registry or external systems (See Fig. 5). The filter applies post process according to the terms and style guide so that the log of pre process procedure is used to make a backward induction and feedback to external systems. This post process procedure maintains consistency in data quality and process control.

The designation of IDs in the registry enables secured information management and access control according to the disclosure class. Agencies and organizations can save time and costs for restructuring each database, and have more flexibility in its design. These functions allow for the coexistence of open protocols and closed schemata designed by each ministry, agency and organization. The frequency of reference to the initial term table should be reduced gradually as new sentences are associated and sentence pairs are accumulated. The standardization efforts and constant maintenance of libraries will be saved. An inquiry made by the citizen will be responded in a consistent manner across agencies due to the existence of style guide and pre- and post-process functions in the filter, and the quality of the presentation of services via the one-stop portal will be improved.

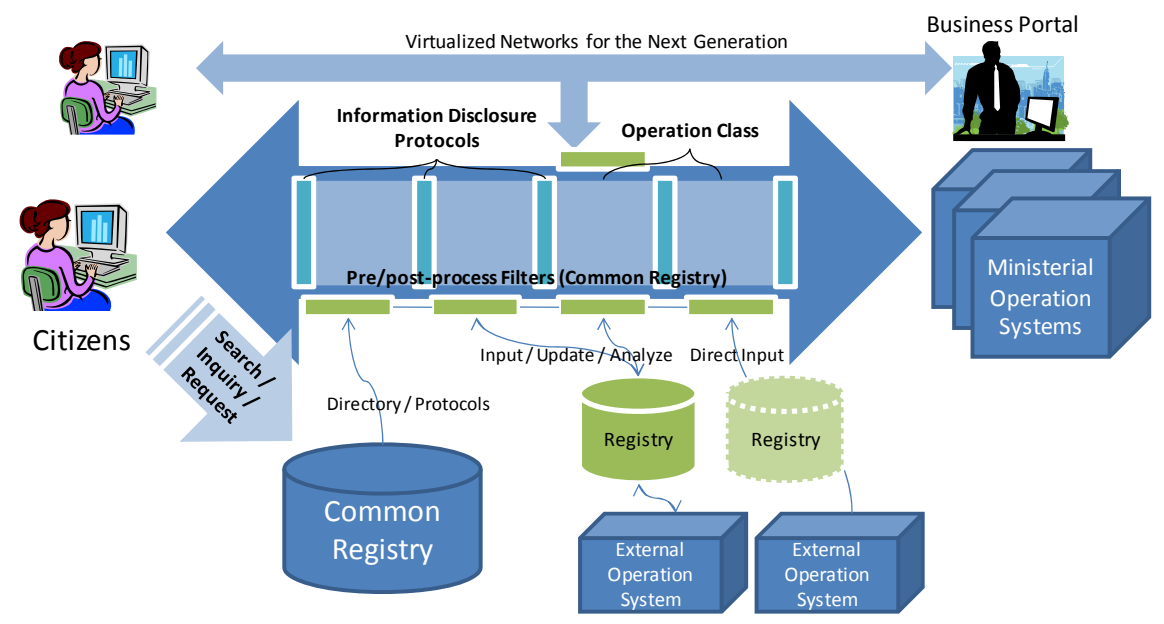

Fig. 5. Pre- and Post-process Filters

\section{Organizational Aspects for the One-Stop Portal}

Japanese government has focused on the optimization of public organizations and the improvement of public services in the e-Gov initiatives. Known and anticipated bottlenecks, as described in [23], exist particularly in the assignment of control and progress monitoring roles in the achievement of loose-coupling of databases. For example, the government has been facing a serious issue with matching personal information for the provision of public pension due to the lack of coordinated databases. To provide citizens with appropriate public pension services, the government should coordinate multiple kinds of personal information such as individuals' past occupational history, 
income, and insurance record. The contribution of this paper is to have proposed a technical solution to break down the organizational silos and introduce the concept of loose-coupling of databases via the registry and directory. Another known issue is related to personal information disclosure. The government is currently discussing institutional issues and possible organizational solutions. One solution will be the establishment of third-party organizations to monitor the use of personal information, and to handle claims so that confidentiality is protected appropriately. In many ways Japan has learned from the case of EU.

Meanwhile, Japanese government has presented a roadmap to implement the one-stop portal starting from central ministries, and then local governments. The portal is going to be implemented into quasi-public sectors, such as utility companies, in which e-Gov system must be able to handle transactions for financial settlements, and tax return. To overcome the known and potential organizational issues, real-life instances should be incorporated thoroughly. In this regard, this paper emphasizes on using standardized XML and enhancing 'reasoning' capability to structure meta-data sets and relationships of service scenarios, in which 'data integrity' is more focused in each business operation system owned by ministries and agencies. It is important to note that, whether or not a pre-defined ontology is prepared i.e. an event-driven service-oriented architecture (EDSOA) [24], which affects the entire government systems, its implementation timing and the balance of XML-based schema sets should be carefully decided when we deal with diversified services. At this point, some features based on ontology and natural language processing functionality, which are to call from relational databases or XML-based databases, are conceptualized in the process filter to supplement the standardized XML schema and handle complex real-life scenarios. As use cases will be collected and studied, then, the varying meaning of data elements and their ambiguity should be assessed to reorganize the meta-data sets based on probability and statistics features to be built in the filters.

\section{Conclusion}

This paper discusses the objectives of Japan's e-Gov policy for the next generation, the architecture of one-stop service platform, and the process of back-office linkages as an evolutionary model. The establishment of networks is an imperative component of e-Gov strategy, and represents the importance of the transition to innovative and transformative e-Gov. To provide citizens with expertise knowledge through e-Gov infrastructure to achieve service innovation, administrative agencies may face challenges in building cooperation and partnerships with private firms because of knowledge and technological intensity inherited in such services. Therefore, it is important to loosely couple databases based on cloud computing, SOA, and virtualization technologies. To do so, cross reference of administrative information, location, access protocols, and data format should be achieved for existing systems employing different technological specifications. Back offices must be connected with the public service platform consisted of the operation process control, the registry, and the directory. As for the incorporation of the registry, it is proposed to manage information in cost and labor saving manners according to a matrix of operation and disclosure class via the 
pre/post-process filters, which is going to be the world first scenario for the one-stop portal system. These developments can be called service innovation in that they represent a new combination of distinct technological fields, new services, means of delivery, and new social infrastructure. Meanwhile, the proposed architecture has not been fully deployed yet, and we should anticipate organizational issues to arise in the future implementation stage. It is essential to build up use cases in real world situations and device appropriate countermeasures.

\section{Acknowledgements}

This research is supported by 'Development of the Fastest Database Engine for the Era of Very Large Database and Experiment and Evaluation of Strategic Social Services Enabled by the Database Engine' as a part of 'Funding Program for World-Leading Innovative R\&D on Science and Technology (FIRST),' Japan's Cabinet Office and Council for Science and Technology Policy. This work is also a part of 'Info-plosion: Cyber Infrastructure for the Information-explosion Era,' Grant-in-Aid for Scientific Research on Priority Areas, MEXT.

\section{References}

1. Brun, M.H., Lanng, C.: Reducing barriers for e-business in SME's through an open service oriented infrastructure. In: International Conference on Electronic Commerce, pp. 403-410. ACM, New York (2006)

2. Forge, S., Colin, B., Bohlin, E., Cave, M.: A Green Knowledge Society -An ICT policy agenda to 2015 for Europe's future knowledge society. A Study for the Ministry of Enterprise, Energy and Communications, Government Offices of Sweden (2009), http://ec.europa.eu/information_society/eeurope/i2010/ greenknowledgesociety.pdf

3. Niehaves, B., Ortbach, K., Becker, J.: The Demographic Challenge: Aging and Depopulation and their Consequences for E-Gov - A Case Study. In: Hawaii International Conference on System Sciences, pp. 1-8. IEEE Press, Los Alamitos (2009)

4. Strategic Headquarters for the Promotion of an Advanced Information and Telecommunications Network Society: i-Japan Strategy 2015 -Striving to Create a Citizen-Driven, Reassuring \& Vibrant Digital Society, Towards Digital inclusion \& innovation. Report released on July 6, 2009 for Japan Cabinet Office (2009),

http: / /www.kantei.go.jp/foreign/policy/it/

i-JapanStrategy2015_full.pdf

5. Project Team for the Next Generation e-Gov and Public Services: Mid-term Report. Document prepared for the 9th meeting (October 26), IT Strategic Headquarters, Japan Cabinet Office (2009), http: / / www . kantei.go.jp/jp/singi /

it2/nextg/meeting/dai9/siryou4.pdf

6. Sudoh, O., Inoue, S., Nakashima, N.: eService Innovation and Sensor Based Healthcare. In: Oya, M., Uda, R., Yasunobu, C. (eds.) Towards Sustainable Society on Ubiquitous Networks, pp. 1-14. Springer, Boston (2008)

7. Schumpeter, J.A.: The Theory of Economic Development. Translation Publishers (1934) 
8. Forge, S., Blackman, C., Bohlin, E., Cave, M.: A Green Knowledge Society -An ICT policy agenda to 2015 for Europe's future knowledge society. Study for the Ministry of Enterprise, Government Offices of Sweden (2009)

9. Rimal, B.P., Eunmi, C., Lumb, I.: A Taxonomy and Survey of Cloud Computing Systems. In: International Joint Conference on INC, IMS and IDC, pp. 44-51. IEEE Press, Los Alamitos (2009)

10. Liang-Jie, Z., Qun, Z.: CCOA: Cloud Computing Open Architecture. In: International Conference on Web Services, pp. 607-616. IEEE Press, Los Alamitos (2009)

11. Chen, R.: Google: The World's First Information Utility? Business \& Information Systems Engineering 1, 53-61 (2009)

12. Cafarella, M.J.: Web-scale extraction of structured data. ACM SIGMOD Record 37, 55-61 (2009)

13. Ohlman, B., Eriksson, A., Rembarz, R.: What Networking of Information Can Do for Cloud Computing. In: International Workshops on Enabling Technologies: Infrastructures for Collaborative Enterprises, pp. 78-83. IEEE Press, Los Alamitos (2009)

14. Katsaros, D., Mehra, P., Pallis, G., Vakali, A.: Cloud Computing: Distributed Internet Computing for IT and Scientific Research. Internet Computing 13(5), 10-13 (2009)

15. Millard, J., Iversen, J.S.: Reorganisation of Government Back-Offices for Better Electronic Public Services -European good practices (back-office reorganisation). European good practice case studies, Final report to the European Commission 3(6), 363-370 (2004)

16. Fraefel, M., Neuroni, A.C., Riedl, R.: Reflecting the relevance of communication in e-government-projects: two case studies in the field of knowledge management in the Swiss public administration. In: Chun, S.A., Sandoval, R., Regan, P. (eds.) Annual International Conference on Digital Government Research, pp. 180-189. ACM, New York (2009)

17. Hu, X., Wang, K.: Application of Data Warehouse Technology in Data Center Design. In: International Conference on Computational Intelligence and Security, pp. 484-488. IEEE Press, Los Alamitos (2008)

18. Guo, Q., Zheng, H., Li, J., Wang, X.: Design and Implementation of Data Management Center Based on Web Services. In: International Conference on Hybrid Intelligent Systems, vol. 2, pp. 322-326. IEEE Press, Los Alamitos (2009)

19. Zhu, J., Nie, Z., Liu, X., Zhang, B., Wen, J.R.: StatSnowball: a Statistical Approach to Extracting Entity Relationships. In: International World Wide Web Conference, pp. 101-110. ACM, New York (2009)

20. Sheu, P.C.Y., Wang, S., Wang, Q., Hao, K., Paul, R.: Semantic Computing, Cloud Computing, and Semantic Search Engine. In: International Conference on Semantic Computing, pp. 654-657. IEEE Press, Los Alamitos (2009)

21. Ma, H., Schewe, K.D., Thalheim, B.: Modelling and Maintenance of Very Large Database Schemata Using Meta-structures. In: van der Aalst, W., Mylopoulos, J., Sadeh, N.M., Shaw, M.J., Szyperski, C. (eds.) Information Systems: Modeling, Development, and Integration, pp. 17-28. Springer, Heidelberg (2009)

22. Franceschet, M., Gubiani, D., Montanari, A., Piazza, C.: From Entity Relationship to XML Schema: A Graph-Theoretic Approach. In: Hunt, Z.B.E., Unland, M.R.R. (eds.) Database and XML Technologies, pp. 165-179 (2009)

23. Janssen, M., Gortmaker, J., Wagenaar, R.W.: Web Service Orchestration in Public Administration: Challenges, Roles and Growth Stages. Information Systems Management 23(2), 44-55 (2006)

24. Overbeek, S.J., Klievink, B., Janssen, M.: A Flexible, Event-Driven, Service-Oriented Architecture for Orchestrating Service Delivery. IEEE Intelligent Systems 24(5), 31-41 (2009) 